IRRITABLE BOWEL SYNDROME

\title{
Long term benefits of hypnotherapy for irritable bowel syndrome
}

\author{
W M Gonsalkorale, V Miller, A Afzal, P J Whorwell
}

Background and aims: There is now good evidence from several sources that hypnotherapy can relieve the symptoms of irritable bowel syndrome in the short term. However, there is no long term data on its benefits and this information is essential before the technique can be widely recommended. This study aimed to answer this question.

Patients and methods: 204 patients prospectively completed questionnaires scoring symptoms, quality of life, anxiety, and depression before, immediately after, and up to six years following hypnotherapy. All

See end of article for authors' affiliations authors affiliations

Correspondence to: Dr W M Gonsalkorale, Hypnotherapy Unit, Withington Hospital, Nell Lane, Manchester M20 2LR, UK; wgonsalkorale@ compuserve.com

Accepted for publication 23 July 2003 subjects also subjectively assessed the effects of hypnotherapy retrospectively in order to define their "responder status".

Results: $71 \%$ of patients initially responded to therapy. Of these, $81 \%$ maintained their improvement over time while the majority of the remaining $19 \%$ claimed that deterioration of symptoms had only been slight. With respect to symptom scores, all items at follow up were significantly improved on pre-hypnotherapy levels $(p<0.001)$ and showed little change from post-hypnotherapy values. There were no significant differences in the symptom scores between patients assessed at 1,2,3,4, or 5+ years following treatment. Quality of life and anxiety or depression scores were similarly still significantly improved at follow up $(p<0.001)$ but did show some deterioration. Patients also reported a reduction in consultation rates and medication use following the completion of hypnotherapy.

Conclusion: This study demonstrates that the beneficial effects of hypnotherapy appear to last at least five years. Thus it is a viable therapeutic option for the treatment of irritable bowel syndrome.

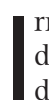
rritable bowel syndrome (IBS) is a functional bowel disorder, the symptoms of which include abdominal pain, distension, and altered bowel habit. Patients also commonly complain of associated extra-colonic symptoms, such as nausea, lethargy, and backache. ${ }^{1}$ This is a common condition and, although the majority of sufferers do not seek medical help, those who do account for up to half of the gastroenterologist's workload. ${ }^{2}{ }^{3}$ Symptoms can be sufficiently severe and troublesome in some individuals as to impair their quality of life. ${ }^{14}$ It is not uncommon for patients to have repeated consultations and investigations and patients tend to consult their own general practitioner for other minor ailments more frequently than other people. ${ }^{5}$ The socioeconomic impact of IBS is therefore considerable and patients account for significant healthcare resources. ${ }^{6}$

Treatment of IBS by conventional means is often unsatisfactory, with symptoms failing to respond to an array of currently available medications. Gut directed hypnotherapy, however, has been shown previously to be extremely effective in the treatment of IBS, with the majority of patients showing improvement in symptoms, associated extra-colonic features and quality of life, ${ }^{7-9}$ findings which have been confirmed by independent studies..$^{10-12}$

Gut directed hypnotherapy comprises a course of up to 12 weekly $\mathrm{l} \mathrm{hr}$ sessions. ${ }^{713}$ Each session consists of induction of the hypnotic state and deepening procedures, followed by "ego strengthening" suggestions relevant to the individual. These are accompanied by further suggestions and interventions, such as inducing warmth in the abdomen using the hands and imagery, directed towards controlling and normalising gut function.

This work led to the establishment of the first hypnotherapy unit in the National Health Service in the UK devoted to the treatment of IBS patients. We have recently published an audit on the first 250 patients treated at this unit, ${ }^{13}$ confirming the beneficial effects of hypnotherapy in a large number of patients. However, outcome was measured immediately after patients completed the course of hypnotherapy. The aim of this present study, therefore, was to establish follow up on a large number of patients treated, to determine the longer term effects of therapy, in terms of symptom improvement, consultation rates, and use of medication.

\section{METHODS}

\section{Patients and procedure}

The following set of questionnaires was mailed to 273 patients with IBS who had completed a course of gut directed hypnotherapy (HT) at least one year previously. The patients were asked to complete and return:

(i) A validated IBS questionnaire, ${ }^{14}$ rating IBS symptoms, extra-colonic features, and quality of life measures by visual analogue scale $(0-100 \mathrm{~mm})$ (Appendix 1);

(ii) The Hospital Anxiety and Depression (HAD) Scale ${ }^{15}$;

(iii) A subjective assessment questionnaire (SAQ), rating the effects of hypnotherapy on symptoms, consultation rates, and use of medication through Likert-type responses, and other information, for example, continued practice of HT techniques (Appendix 2).

All patients had already completed the IBS Questionnaire and HAD Scale both immediately before and after the course

Abbreviations: HAD, Hospital Anxiety and Depression Scale; HT, hypnotherapy; IBS, irritable bowel syndrome; $S A Q$, subjective assessment questionnaire 
of HT, and therefore data were available for three different time points-that is, pre-HT, post-HT, and follow up.

\section{Statistical analysis}

Mean values (with SEM) were calculated for all scores in the IBS questionnaire and HAD Scale completed pre- and postHT and at follow up. Intra-individual differences in scores between these time points, with positive and negative values denoting improvement or deterioration in scores, respectively, relative to the earlier time point, were compared by paired $t$ test. Comparison between individual groups was performed using independent t test (two groups) or analysis of variance (more than two groups). For each item in the SAQ, data are given as the proportion (\%) of patients rating each response category. Intra-individual comparisons were made using McNemar's test and independent groups compared using $\chi^{2}$ test. Spearman $(\rho)$ correlation coefficients were calculated to assess relationships between variables. For further analysis, patients were subdivided into responders and non-responders, based on the response to SAQ(i), with responders defined as patients rating their symptoms either "very much better" or "moderately better" at the end of the course of HT and non-responders as those rating symptoms "slightly better" or less. Patients were also grouped according to the number of years that had lapsed since completing treatment.

Binary logistic regression analysis was performed to identify factors affecting "responder" status. In order to limit the number of variables examined at any one time, a stepwise analysis was carried out using a series of variable blocks, with blocks relating to pre-HT measures for all symptoms, quality of life, and HAD scores as well as age, gender, and bowel habit.

Overall scores for IBS symptoms and extra-colonic features were treated as the primary outcomes. For the purpose of comparing secondary endpoints-that is, the individual measures making up these overall scores-only differences at the $0.1 \%(\mathrm{p}<0.001)$ were interpreted as showing reasonable evidence of a true difference, instead of adjusting for multiple comparisons.

\section{RESULTS}

\section{Return rate}

Of the 273 questionnaires 204 were returned, representing a $75 \%$ return rate. These were reasonably equally distributed according to the time since completing treatment $(1<2$ years ago: $22.4 \% ; 2<3$ years: $18.4 \% ; 3<4$ years: $27.8 \%$; $4<5$ years: $21.4 \%$; $\geqslant 5: 10.0 \%)$.

\section{Subjective assessment questionnaire Improvement after hypnotherapy (SAQ(i))}

There were $106(52.0 \%)$ patients who reported their symptoms as "very much better" at the end of the course of HT, $39(19.1 \%)$ as "moderately better", 32 (15.7\%) as "slightly better", while 27 (13.2\%) reported no change in symptoms. No patients reported worsening of symptoms. Patients in whom symptoms were very much or moderately better $(71.1 \%$ of total) were then defined as "responders", and those with only slight improvement or no change as "non-responders".

Progress in symptoms since completing HT (SAQ(ii)) Subdividing according to responder status as above, $81.3 \%$ of responders maintained the improvement gained, with the majority stating that their symptoms had improved even further (29.2\% improved "much more", 24.3\% "moderately more", $11.1 \%$ "slightly more", and $16.7 \%$ "about the same as

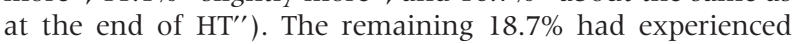
mostly slight deterioration in symptoms. More responders than non-responders stated that symptoms had continued to improve after finishing HT (responders (R) $v$ non-responders (NR): $64.6 \%$ v $35.0 \%, \chi^{2}=13.54, \mathrm{p}<0.001$ ) and symptom improvement was mainly rated as slight for these nonresponders. The majority of non-responders, therefore, had no improvement in symptoms during the follow up period, with $49.1 \%$ being about the same as at the end of treatment (that is, about the same or only slightly improved compared with before HT) and 15.8\% slightly worse.

\section{IBS questionnaires \\ IBS symptoms}

Scores from pre- and post-HT IBS questionnaires were compared in patients returning and not returning follow up questionnaires, in order to ensure that those returning these questionnaires were representative of the patient group as a whole. No differences in symptom severity before treatment or in initial improvement after HT emerged. Questionnaires returned $(\mathrm{n}=204) v$ not returned $(\mathrm{n}=69)$ : pre-HT (mean, SEM): pain severity 56.1 (1.9) $v 60.1$ (2.6); pain frequency 55.0 (2.4) v 64.1 (3.3); bloating 61.0 (1.9) v 61.8 (2.9); bowel habit dissatisfaction $71.6(1.8) \vee 73.0$ (2.4); life interference 72.5 (1.4) v 75.1 (1.7); overall score 314.2 (6.1) v 330.4 (8.6), all $\mathrm{p}>0.05$. Pre-HT/post-HT intra-individual differences (mean, 95\% confidence interval (CI)): pain severity 25.5 (21.5 to 29.5$) v 27.2$ (22.0 to 32.4); pain frequency 23.8 (19.1 to 28.6$) v 35.3$ (28.5 to 42.1 ); bloating 29.4 (25.5 to 33.4) $v$ 31.9 (26.7 to 37.2 ); life interference 33.2 (29.4 to 36.9) $v 31.1$ (25.7 to 36.6); overall score 144.1 (128.8 to 159.4) $v 151.3$ (129.2 to 173.5 ), all $\mathrm{p}>0.05$, except pain frequency where $\mathrm{p}=0.005$.

Intra-individual differences in scores between the three different time points (that is, pre-HT, post-HT, and follow up) in the 204 patients for whom follow up data were obtained (figure 1) showed that all symptoms as well as the overall score had improved immediately after HT and remained better at follow up (pre-HT/post-HT and pre-HT/follow up intra-individual differences, all $\mathrm{p}<0.001)$. Symptoms had increased slightly at follow up compared with post-HT levels but this was significant only for bloating and bowel habit

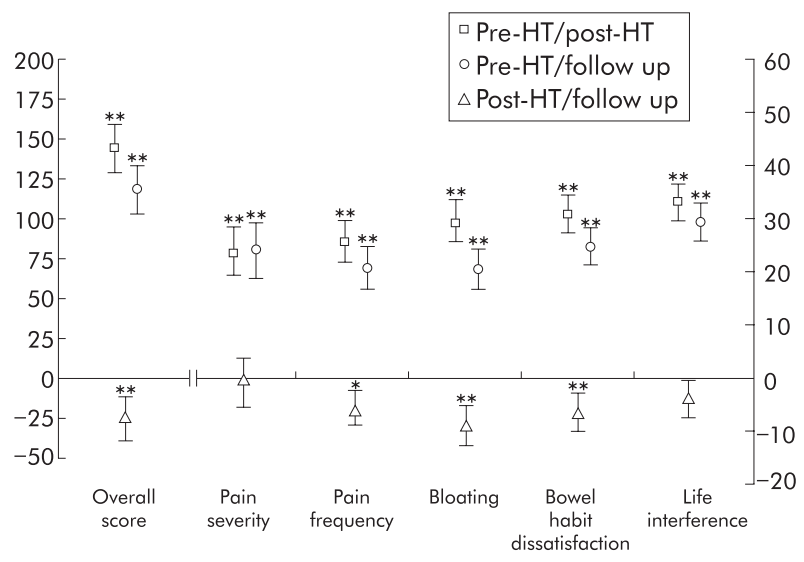

Figure 1 Intra-individual differences in IBS symptom scores for all patients $(n=204)$ between the different time points - that is, pre-HT post-HT, pre-HT/follow up, and post-HT/follow up. Data expressed as mean $(95 \%$ confidence interval (CI)), with positive and negative values denoting reduction (improvement) and increase (deterioration) in score relative to earlier time point. (Mean values of scores at these time points are given in the text.) Overall score treated as the primary outcome and therefore no adjustment is made for multiple comparisons. "Unadjusted" comparisons for individual symptoms significant at the $0.1 \%$ level are assumed significant at the $5 \%$ level if multiple comparison adjustment is made. ${ }^{*} p<0.01,{ }^{* *} p<0.001$. 
dissatisfaction (post-HT/follow up intra-individual differences, both $\mathrm{p}<0.001$ ).

Patients' rating of improvement in the SAQ was reflected in the IBS questionnaire scores with a direct correlation, firstly, between rating of improvement after HT, SAQ(i), and intra-individual differences in pre-HT/post-HT overall IBS scores $(\rho=0.433, \mathrm{p}<0.001)$, and, secondly, between rating of symptom progress since completing HT, SAQ(ii), and differences in post-HT/follow up overall IBS scores $(\rho=0.488, \mathbf{p}<0.001)$.

\section{Analysis according to responder status}

As shown in table 1 , severity of IBS symptoms prior to HT was no different between the responders and non-responders ( $\mathrm{R} v \mathrm{NR}$ : all $\mathrm{p}>0.05$ ). However, mean scores for responders were significantly lower than those for non-responders both immediately after HT (all $\mathrm{p}<0.001$ ) and at follow up (all $\mathrm{p}<0.001$, except pain severity $(\mathrm{p}<0.5)$ and bloating $(p<0.01)$. Intra-individual differences in scores between the different time points (figure 2) shows that while some symptom measures were also significantly improved in the non-responder group post-HT and at follow up (bowel habit dissatisfaction and life interference $\mathrm{p}<0.001$ at both time points), these changes were small compared with those in responders. Indeed, responders had greater reduction in symptom scores than non-responders both immediately after HT (pre-HT/post-HT intra-individual differences, R $v$ NR: all $\mathrm{p}<0.001$ ) and at follow up (pre-HT/follow up intraindividual differences, $\mathrm{R} v \mathrm{NR}$ : all $\mathrm{p}<0.001$ except pain frequency, $p<0.005)$. Furthermore, no significant intraindividual differences between post-HT and follow up symptom scores occurred for non-responders (all p>0.05), showing that symptoms did not improve with the course of time.

\section{Analysis according to time lapsed since completing treatment}

There was no association between the time, in years, since responders had completed treatment and intra-individual differences in post-HT and follow up overall IBS score ( $\rho=-0.027, \mathrm{p}=0.752)$, suggesting that those with a longer gap since treatment had maintained improvement just as well as those who had finished more recently. In addition, there were no marked differences in overall IBS scores (table 2) between the different time intervals either before HT $(p=0.310)$ or at follow up $(p=0.458)$ or in the pre-HT/ follow up intra-individual differences in this score $(\mathrm{p}=0.781)$.

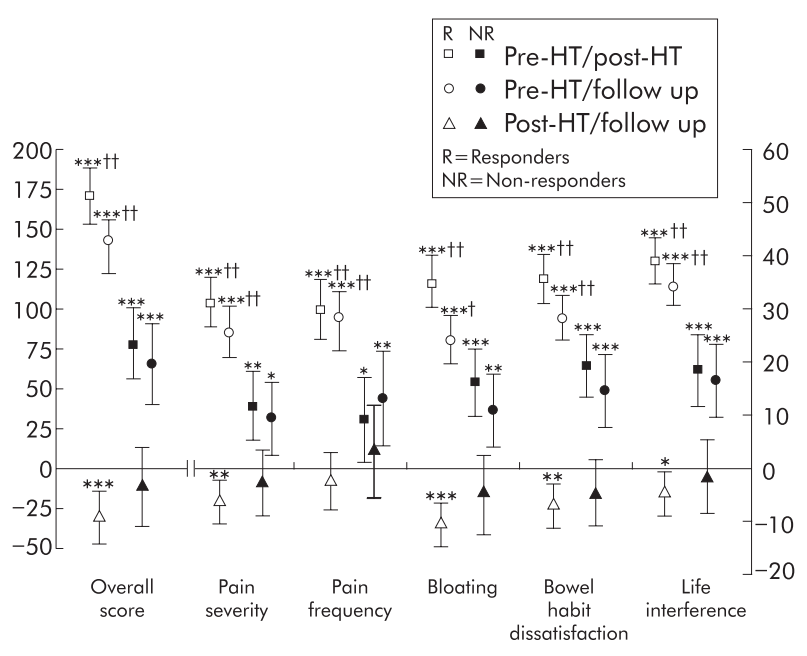

Figure 2 Intra-individual differences in IBS symptom scores for responders and non-responders between the different time points shown. Data expressed as mean $(95 \%$ confidence interval (CI)), with positive and negative values denoting reduction and increase in score relative to earlier time point. (Mean values of scores at these time points are given in table 1.) Overall score treated as the primary outcome and therefore no adjustment is made for multiple comparisons. "Unadjusted" comparison for individual symptoms significant at the $0.1 \%$ level are assumed to be significant at the $5 \%$ level if multiple comparison adjustment made. ${ }^{*} p<0.05,{ }^{* *} p<0.01,{ }^{* * *} p<0.001$ (intra-individual difference); ${ }^{+} p<0.005,{ }^{+} p<0.001$ (responders $v$ non-responders).

\section{Extra-colonic symptoms}

Severity of extra-colonic symptoms before HT was similar for both responders and non-responders (table 3). Responders had significant improvement in individual symptoms and overall score after HT (pre-HT/post-HT intra-individual differences, all $\mathrm{p}<0.001$ ) and most of these remained better than pre-HT levels at follow up (pre-HT/follow up intraindividual differences all $\mathrm{p}<0.001$, except urinary symptoms, thigh pain, and bodily aches, $\mathrm{p}<0.05)$. Although nonresponders had significant improvement in overall extracolonic score and some individual symptoms immediately after HT (pre-HT/post-HT intra-individual differences: overall score, nausea, excess wind, lethargy all $\mathrm{p}<0.001$ ), this had deteriorated at follow up. Responders had more pronounced improvement in overall score than non-responders, both post-HT and at follow up (intra-individual differences, R $v$ NR: pre-HT/post-HT $\mathrm{p}<0.001$; pre-HT/follow up: $\mathrm{p}<0.005$ ) and for a number of individual symptoms after HT, including excess wind (pre-HT/follow up intra-individual differences, $\mathrm{R} v \mathrm{NR}$ : $\mathrm{p}<0.001)$, lethargy, backache, and

Table 1 IBS symptom scores

\begin{tabular}{|c|c|c|c|c|c|c|}
\hline \multirow[b]{2}{*}{ Symptom } & \multicolumn{2}{|l|}{ Pre-HT } & \multicolumn{2}{|l|}{ Post-HT } & \multicolumn{2}{|l|}{ Follow up } \\
\hline & $\mathbf{R}$ & NR & $\mathbf{R}$ & NR & $\mathbf{R}$ & NR \\
\hline Pain severity & $58.0(2.2) \dagger$ & $51.4(3.6)$ & $26.4(1.9)^{* * *}$ & $39.9(3.3)$ & $32.2(2.1)^{*}$ & $42.0(3.6)$ \\
\hline Pain frequency & $53.1(2.8) \dagger$ & $59.3(4.5)$ & $22.8(2.6)^{\star \star \star}$ & $50.2(4.6)$ & $25.2(2.4)^{* \star *}$ & $45.7(5.1)$ \\
\hline Bloating & $61.0(2.2) \dagger$ & $60.8(3.8)$ & $27.1(2.1)^{* * *}$ & $44.0(4.1)$ & $36.9(2.1)^{* *}$ & $49.0(4.2)$ \\
\hline $\mathrm{BH}$ dissatisfaction & $71.1(2.1) \dagger$ & $72.6(3.4)$ & $35.6(1.8)^{\star \star *}$ & $53.9(3.4)$ & $42.5(1.8)^{\text {***}}$ & $58.0(3.7)$ \\
\hline Life interference & $71.4(1.6) \dagger$ & $75.2(2.7)$ & $32.2(1.9)^{\star \star \star}$ & $57.1(3.1)$ & $37.0(1.9)^{* * *}$ & $58.4(3.3)$ \\
\hline Overall score & $312.2(7.4) \dagger$ & $318.2(11.0)$ & $143.6(8.3)^{\star * *}$ & $241.7(13.1)$ & $173.7(8.0)^{\text {** }}$ & * 251.3 (14.8) \\
\hline
\end{tabular}

Irritable bowel syndrome (IBS) symptom scores (mean, SEM) shown for responders (R) and non-responders (NR), as measured in IBS questionnaire (VAS 0-100 mm), completed at the time points shown. For data in this table (and similarly in all tables), overall score is treated as the primary outcome and therefore no adjustments are made for multiple comparisons. "Unadjusted" comparisons for individual symptoms significant at the $0.1 \%$ level $(p<0.001)$ assumed significant at the $5 \%$ level if multiple comparison adjustments were made. ${ }^{*} \mathrm{p}<0.05 ;{ }^{* \star} \mathrm{p}<0.01 ;{ }^{* * *} \mathrm{p}<0.001 ; \mathrm{tp}>0.05$ (R $\left.v \mathrm{NR}\right)$

$\mathrm{BH}$, bowel habit; HT, hypnotherapy. 
Table 2 Overall IBS scores for responders according to time since treatment

\begin{tabular}{lllll}
\hline $\begin{array}{l}\text { Years } \\
\text { since } \\
\text { HT }\end{array}$ & N & Pre-HT* & Follow up* & Pre-HT/follow upt \\
\hline $1<2$ & 37 & $283.8(13.9) \ddagger$ & $161.2(16.1) \S$ & $122.7(95.5$ to 149.8$)$ ศ \\
$2<3$ & 25 & $330.8(18.2)$ & $197.6(21.4)$ & $133.2(94.3$ to 172.1$)$ \\
$3<4$ & 36 & $328.4(14.1)$ & $183.8(16.8)$ & $145.1(105.8$ to 184.5$)$ \\
$4<5$ & 32 & $319.5(16.6)$ & $168.5(16.9)$ & $151.0(107.5$ to 194.5$)$ \\
$\geqslant 5$ & 15 & $298.8(23.3)$ & $147.5(18.0)$ & $151.3(93.1$ to 209.6$)$
\end{tabular}

Data shown as: *overall IBS scores (mean (SEM)) and tintra-individual differences (mean, $95 \% \mathrm{Cl}$ ) for time points shown for responders, subdivided according to time since completing treatment. Intra-individual differences having positive value denote reduction (improvement) in score relative to earlier time point.

$\neq p=0.310 ; \S p=0.458 ; \uparrow p=0.781$ (all ANOVA).

heartburn (all $\mathrm{p}<0.005$ ), although this was not evident at follow up (intra-individual pre-HT/follow up differences, $\mathrm{R} v$ NR: excess wind, lethargy, backache all $\mathrm{p}<0.05$; heartburn $\mathrm{p}>0.05$ )

\section{Quality of life, anxiety, and depression}

Quality of life measures before HT were no different for responders and non-responders (table 4). Although there was some improvement in the non-responder group immediately after HT (pre-HT/post-HT intra-individual differences, all $\mathrm{p}<0.005$, physical well being and control $\mathrm{p}<0.001$ ), this was significant at follow up only for mood $(p<0.005)$. Improvement occurred in responders both after HT and at follow up compared with pre-HT levels (intra-individual differences: pre-HT/post-HT all $\mathrm{p}<0.001$; pre-HT/follow up all $\mathrm{p}<0.001$, social/relationships $\mathrm{p}<0.005$ ) and this was more marked than in non-responders, particularly for psychic and physical well being ( $\mathrm{R} v$ NR: pre-HT/post-HT $\mathrm{p}<0.001$; preHT/follow up $\mathrm{p}<0.01$ ).

HAD scores for anxiety and depression were similar in both patient groups before HT (R $v$ NR: HAD "A" anxiety (mean $(\mathrm{SEM}))$ : 10.7 (0.4) v 9.8 (0.6), $\mathrm{p}=0.184$; HAD “D” depression: $6.7(0.3) v 6.8(0.4), \mathrm{p}=0.798)$. Scores had improved in both groups post-HT and were still better than pre-HT levels at follow up (intra-individual differences (mean (95\%CI)): pre-HT/post-HT: anxiety, R: 4.1 (3.5 to 4.7$),$ NR: 2.4 (1.4 to 3.3); depression, R: 3.4 (2.8 to 4.0 ), NR: 1.7 (0.9 to 2.6), all $\mathrm{p}<0.001$; pre-HT/follow up: anxiety, R: 2.7 (2.0 to 3.3), $\mathrm{p}<0.001$; NR: 1.5 (0.5 to 2.5), $\mathrm{p}=0.003$; depression, R: 2.5 (1.9 to 3.2), NR: $1.4(0.7$ to 2.2$)$, both $\mathrm{p}<0.001)$. Improvement was overall greater in the responder group, particularly post-HT (R $v$ NR: anxiety and depression, both $\mathrm{p}=0.002)$ but less evident at follow up ( $\mathrm{R} v \mathrm{NR}$ : anxiety: $\mathrm{p}=0.068$; depression, $\mathrm{p}=0.031)$. Fewer responders were significantly anxious or depressed (HAD scores $>9$ ) after HT and at follow up, while fewer non-responders were anxious post-HT only (HAD "A" $>9$ (anxious): Pre-HT $v$ post-HT: R: $59.0 \%$ v $24.4 \%, \mathrm{p}<0.001$; NR: $48.3 \%$ v $28.1 \%, \mathrm{p}=0.008$; versus follow up: R: $31.9 \%, \mathrm{p}<0.001$; NR: $v 37.3 \%, \mathrm{p}=0.210$; HAD "D" $>9$ (depressed): Pre-HT $v$ post-HT: R: $20.7 \%$ v 5.5\%, $\mathrm{p}<0.001$; NR: $18.6 \%$ v $11.9 \%, \mathrm{p}=0.424$; versus follow up: R: 9.0\%, $\mathrm{p}=0.002 ; \mathrm{NR}: 13.6 \%, \mathrm{p}=0.549$, McNemar's test).

\section{Consultation rates}

The majority of responders compared with around half of non-responders stated they had seen their GP and the hospital consultant less often about IBS symptoms since HT, and more responders had seen their GP less often about other symptoms (consulted "less often", "about same", "more often": R $v$ NR (\%): GP/IBS symptoms: $87.9 \%, 10.7 \%, 1.4 \% v$ $50.9 \%, 45.6 \%, 3.5 \%, \chi^{2}=31.75, \mathrm{p}<0.001$; Consultant/IBS symptoms: $83.3 \%, 13.7 \%, 3.7 \%$ v $54.8 \%, 40.5 \%, 4.8 \%$, $\chi^{2}=13.41, \mathrm{p}=0.001 ; \mathrm{GP} / \mathrm{other}$ symptoms: $61.2 \%, 32.6 \%$, $\left.6.2 \% \vee 21.8 \%, 63.6 \%, 14.5 \%, \chi^{2}=24.10, \mathrm{p}<0.001\right)$.

\section{Use of medication}

Medication taken before HT included antispasmodics (42.0\% of all patients on medication), antidiarrhoeals (23.2\%), laxatives (12.3\%), bulking agents (9.4\%), antidepressants $(21.0 \%)$, and anxiolytics (10.9\%), with $27.5 \%$ taking at least two types of medication. A similar proportion of responders and non-responders were taking medication before HT but fewer responders were on medication during follow up ( $\mathrm{R} v$ NR: Pre-HT: $67.6 \% \quad v 67.8 \%, \chi^{2}=0.03, \mathrm{p}=0.856$; during follow up: $36.6 \% \vee 55.9 \%, \chi^{2}=6.27, p=0.012$ ). Of those patients continuing to take medication, more responders stated they had taken it less often than before ("less often", "about same", "more often", R v NR: 62.3\%, 26.4\%, $11.3 \% v$ 9.1\%, 69.7\%, 21.2\%, $\left.\chi^{2}=23.91, \mathrm{p}<0.001\right)$.

While proportionately more non-responders than responders with low anxiety and depression scores (HAD scores $<10)$ at follow up were taking antidepressants and/or anxiolytics (NR $v$ R: $21.1 \% \vee 6.0 \%, \mathrm{p}<0.001$ ) these represented only a minority of non-responders with low scores, since $63 \%$ and $80 \%$ of this group had low HAD " $\mathrm{A}$ " and " $\mathrm{D}$ " scores, respectively.

\section{Factors influencing initial response to hypnotherapy}

There were no differences between responders and nonresponders in any baseline measures of symptoms, quality of life, or HAD scores (tables 1,3 , and 4 and HAD results section) but more males than females were non-responders

Table 3 Extra-colonic symptom scores

\begin{tabular}{|c|c|c|c|c|c|c|}
\hline \multirow[b]{2}{*}{ Symptoms } & \multicolumn{2}{|l|}{ Pre-HT§ } & \multicolumn{2}{|l|}{ Pre-HT/Post-HT } & \multicolumn{2}{|l|}{ Pre-HT/follow up ब } \\
\hline & $\mathbf{R}$ & NR & $\mathbf{R}$ & NR & $\mathbf{R}$ & NR \\
\hline Nausea/vomiting & $28.5(2.3) \ddagger$ & $27.8(3.8)$ & $13.9(10.4 \text { to } 17.4)^{\star \star \star}$ & $11.4(6.1 \text { to } 16.7)^{* * *}$ & $13.0(9.5 \text { to } 16.5)^{\star * *}$ & $11.5(4.9 \text { to } 18.0)^{* *}$ \\
\hline Early satiety & $29.1(2.3) \ddagger$ & $23.8(3.8)$ & $10.1(6.8 \text { to } 13.4)^{* * *}$ & $6.5(1.8 \text { to } 11.2)^{\star *}$ & $10.1(5.8 \text { to } 14.5)^{\star * *}$ & $6.9(2.3 \text { to } 11.5)^{* *}$ \\
\hline Heartburn & $31.7(2.4) \ddagger$ & 26.5 (3.9) & $15.9(11.7 \text { to } 20.2)^{* * *}+\dagger$ & $5.1(-0.9$ to 11.1$)$ & $10.5(5.9 \text { to } 15.2)^{\star \star *}$ & $7.8(1.9 \text { to } 13.7)^{*}$ \\
\hline Backache & $49.7(2.6) \ddagger$ & $41.6(4.2)$ & $16.9(12.8 \text { to } 21.1)^{* * *}+\dagger$ & $4.5(-1.7$ to 10.7$)$ & $14.2(9.7 \text { to } 18.6)^{* * *} \dagger$ & $3.7(-3.7$ to 11.0$)$ \\
\hline Headaches & $41.6(2.4) \ddagger$ & $35.7(4.0)$ & $13.3(9.6 \text { to } 17.0)^{* * *}$ & $8.9(3.5 \text { to } 14.3)^{* *}$ & $9.4(5.6 \text { to } 13.2)^{* * *}$ & $3.9(-1.4$ to 9.2$)$ \\
\hline Excess wind & $74.2(1.9) \ddagger$ & $74.6(3.5)$ & $33.7(28.9 \text { to } 38.5)^{\star * \star}$ t†† & $19.2(13.4 \text { to } 25.0)^{* * *}$ & $18.7(14.1 \text { to } 23.3)^{* * *} \dagger$ & $9.6(2.5 \text { to } 16.6)^{* *}$ \\
\hline Lethargy & $67.2(2.3) \ddagger$ & $67.2(3.6)$ & $29.0(24.5 \text { to } 33.5)^{* * *}+\dagger$ & $15.1(7.7 \text { to } 22.5)^{* \star *}$ & $16.4(11.8 \text { to } 21.0)^{\star * *} \dagger$ & $5.4(-1.9$ to 12.6$)$ \\
\hline Urinary & $48.0(2.8) \ddagger$ & $38.7(4.4)$ & $15.9(11.5 \text { to } 20.4)^{* * *}$ t† & $2.2(-5.1$ to 9.4$)$ & $6.2(1.1 \text { to } 11.3)^{*}+$ & $-4.6(-13.8$ to 4.5$)$ \\
\hline Thigh pain & $24.3(2.5) \pm$ & $24.9(4.6)$ & $10.3(6.1 \text { to } 14.5)^{* * *}$ & $7.6(1.1 \text { to } 14.1)^{*}$ & $4.5(-0.5 \text { to } 9.4)^{*}$ & $3.2(-4.4,10.8)$ \\
\hline Bodily aches & $47.9(2.7) \ddagger$ & $46.4(4.6)$ & $16.6(12.0 \text { to } 21.3)^{\star * *} \dagger$ & $6.9(-0.6$ to 13.9$)$ & $6.5(1.4 \text { to } 11.6)^{*}$ & $0.4(-7.2$ to 8.0$)$ \\
\hline Overall score & $219.5(7.6) \ddagger$ & $201.1(11.5)$ & $80.4(65.1 \text { to } 95.6)^{* * *}+\dagger \dagger$ & $40.1(24.5 \text { to } 55.6)^{\star * *}$ & $53.7(41.1 \text { to } 66.2)^{\star * *}+\dagger$ & $21.6(7.6 \text { to } 35.5)^{\star *}$ \\
\hline
\end{tabular}

Data shown for responders (R) and non-responders (NR) as §mean scores (SEM); - intra-individual differences (mean (95\% CI)) between time points shown, with positive and negative values denoting reduction and increase in scores, respectively, relative to earlier time point.

${ }^{*} \mathrm{p}<0.05,{ }^{* *} \mathrm{p}<0.005,{ }^{* * *} \mathrm{p}<0.001$ (versus pre-HT); $\dagger+\uparrow \mathrm{p}<0.001 ; \dagger+\mathrm{p}<0.01 ; \uparrow \mathrm{p}<0.05 ; \mathrm{fp}>0.05$ (R $v \mathrm{NR}$ ). 
Table 4 Quality of life measures

\begin{tabular}{|c|c|c|c|c|c|c|}
\hline & \multicolumn{2}{|l|}{ Pre-HT§ } & \multicolumn{2}{|l|}{ Pre-HT/Post-HTब } & \multicolumn{2}{|l|}{ Pre-HT/follow up ब } \\
\hline & $\mathbf{R}$ & NR & $\mathbf{R}$ & NR & $\mathbf{R}$ & NR \\
\hline Psychic well being & $50.3(1.8) \ddagger \ddagger$ & $55.1(3.1)$ & $27.6(23.9 \text { to } 31.4)^{* * *}+\dagger \dagger$ & $10.3(4.4 \text { to } 16.2)^{* *}$ & $18.7(14.2 \text { to } 23.2)^{* * *}+\dagger$ & $6.2(-0.4$ to 12.8$) \ddagger$ \\
\hline Physical well being & 47.2 (1.7)市 & $45.1(2.7)$ & 21.8 (18.6 to 25.0$)$ *** $+\dagger \dagger$ & $12.0(7.4 \text { to } 16.6)^{* * *}$ & $12.5(8.9 \text { to } 16.0)^{* * *} \dagger$ & $4.9(-0.1$ to 10.0$) \ddagger$ \\
\hline Mood & 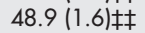 & $47.4(2.6)$ & $19.9(16.4 \text { to } 23.4)^{* * *}$ t† & $10.6(4.4 \text { to } 16.8)^{* *}$ & $12.8(9.0 \text { to } 16.5)^{\star * *}$ 㧊 & $10.2(4.2 \text { to } 16.3)^{* *}$ \\
\hline Control & 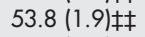 & $59.2(3.4)$ & $18.2(14.8 \text { to } 21.5)^{\star \star *} \dagger$ & $11.0(5.2 \text { to } 16.8)^{\star \star *}$ & $13.0(8.8 \text { to } 17.3)^{* \star \star} \dagger$ & $4.7(-2.5$ to 11.8$) \ddagger$ \\
\hline Social/relationships & $68.6(1.6) \pm \ddagger$ & $67.1(2.9)$ & $10.8(8.6 \text { to } 18.6)^{* * *}$ t† & $5.0(1.7 \text { to } 8.3)^{\star *}$ & $7.4(4.3 \text { to } 10.4)^{*} \neq \ddagger$ & $3.9(-1.3$ to 7.6$) \neq$ \\
\hline
\end{tabular}

Data shown for responders (R) and non-responders (NR) as §mean scores (SEM) and 9 intra-individual differences (mean (95\% CI)) between time points. Note: In contrast with other symptom scores, higher values in quality of life measures signify improvement; positive and negative values in intra-individual differences denote reduction (deterioration) and increase (improvement) in scores, respectively, relative to earlier time point.

${ }^{*} \mathrm{p}<0.05 ;{ }^{* *} \mathrm{p}<0.005 ;{ }^{* * *} \mathrm{p}<0.001 ; \mathrm{fp}>0.05$ (versus pre-HT); $\uparrow+\uparrow \mathrm{p}<0.001 ; \dagger+\mathrm{p}<0.01 ; \mathrm{tp}<0.05 ; \neq \neq \mathrm{p}>0.05$ (R vNR).

(males $v$ females: R: $57.5 \% \vee 74.7 \%$; NR: $42.5 \% \vee 25.3 \%$, $\left.\chi^{2}=4.63, \mathrm{p}=0.031\right)$. Although more males had diarrhoea predominant bowel habit (males $v$ females, bowel habit type: constipation $12.8 \% \vee 25.5 \%$, diarrhoea $51.3 \% \quad v \quad 24.8 \%$, alternating $\left.35.9 \% \vee 49.7 \%, \chi^{2}=10.77, \mathrm{p}=0.005\right)$, no more non-responders were found with this or any other bowel habit type, either for patients as a whole or for males (constipation $v$ diarrhoea $v$ alternating: all patients, R: 69.6\% $v 73.3 \%$ v 71.3\%; NR $30.4 \%$ v $26.7 \%$ v $28.7 \%, \chi^{2}=0.19$, $\mathrm{p}=0.911$; males: R: $40.0 \%$ v $60.0 \%$ v 57.1\%; NR: $60.0 \% v$ $\left.40.0 \% \vee 42.9 \%, \chi^{2}=0.66, p=0.721\right)$. Non-responders were also overall slightly older than responders (mean age (SEM), years: R $v$ NR: 45.1 (1.2) v 50.3 (1.8), $\mathrm{p}=0.017$ ). However, binary logistic regression analysis identified only gender as having a significant relationship with responder status (OR (95\% CI): 0.30 (0.16 to 0.97$), p=0.042)$.

\section{Continued practice of hypnotherapy techniques during follow up}

More responders than non-responders continued to practise HT techniques after treatment ( $\mathrm{R}$ (\% patients): $85 \%$ v 58\%, $\mathrm{p}<0.001)$, although both groups had a similar pattern of practice (R $v$ NR: with tape only: $38.7 \%$ v 36.4\%; without tape: $42.4 \%$ v 42.5\%; both with/without tape: $18.9 \%$ v $21.1 \%$; $\left.\chi^{2}=0.24, \mathrm{p}=0.970\right)$. There was no difference in symptom change during follow up between patients who still practised and those who did not (intra-individual difference in postHT/follow up overall IBS score (mean (SEM)), practice $v$ no practice: all patients: $-19.5(16.6) v-27.4$ (7.8), $\mathrm{p}=0.392$; $\mathrm{R}$ only: -24.8 (32.6) $v-31.9(8.7), \mathrm{p}=0.628)$. Similarly, there was no correlation between symptom change and frequency of practice (all patients: $\rho=0.027, \mathrm{p}=0.777$; $\mathrm{R}$ only: $\rho=0.067, \mathrm{p}=0.572$ ).

\section{Other considerations}

The continued improvement seen in responders cannot be explained by the use of other treatments after finishing HT. Only $14(9.7 \%)$ of responders had tried other treatments, including dietary changes, alternative medicines, yoga, and reflexology and found these helpful. Of all patients, 93.1\% considered that the course of HT had been worthwhile, which included all of the responders and $76.3 \%$ of non-responders.

\section{DISCUSSION}

This study presents the first long term follow up of a large number of patients who have undergone hypnotherapy for IBS symptoms. Immediately after HT, $71 \%$ of patients considered their symptoms very much or moderately better and these patients were defined as "responders". Of these initial responders, $81 \%$ maintained the benefit of treatment or reported further improvement. In contrast, non-responders, with only slight or no improvement in symptoms with HT, had little or no change in symptoms over the follow up period.
There was a good correlation between the SAQ ratings and the objective symptom scores. IBS symptom scores were significantly improved after HT and remained so at follow up, with only a slight deterioration compared with post-HT levels. The beneficial effects did not appear to decline with time since patients who had finished treatment more than five years ago maintained symptom improvement just as well as those who had completed only a year ago. Similarly, extracolonic features, quality of life, anxiety, and depression scores were still better at follow up compared with pre-HT levels. Interestingly, all patients reported taking less medication and had consulted less often for IBS and other symptoms, but this was particularly striking for the responder group.

The subdivision of patients into "responders" and "nonresponders", based on the SAQ rating of symptom change, was undertaken in order to concur with the current practice of using a subjective global assessment as a primary outcome in clinical trials in functional bowel disorders. ${ }^{16}$ It also facilitated further analysis of the data, and indeed revealed that there were clear differences between responders and non-responders in terms of IBS and other symptom scores, consultation rates, and use of medication after treatment.

The SAQ was only completed at follow up, hence requiring patients to assess the effects of HT retrospectively and therefore the reliability of their reports could be called into question. However, the fact that the SAQ correlated with changes in symptom rating from the IBS questionnaires would indicate that the SAQ assessments are a reliable reflection of treatment outcome. Similarly, patients' reports of consultation rates and use of medication must be interpreted with some caution. However, one could determine the number of patients who had stopped medication after HT, since medication was recorded at the start of HT and at follow up. Reports in terms of fewer consultations and less medication were markedly different between responders and non-responders, suggesting that these cannot be explained entirely by recall bias.

The return rate in this study was $75 \%$. These patients can be considered to be representative of all patients contacted since their pre-HT IBS symptom scores and improvement immediately after HT were similar to the patients who did not return the follow up questionnaires. This study was an audit of patients who had undergone hypnotherapy and hence there was no control group, which may be considered a weakness to the study, although it is difficult to conceive how one could have been included. There is known to be a high level of placebo response in IBS patients which can last for at least three months. ${ }^{17}$ However, the improvement seen in this study lasted at least one year and was at the same level in patients after five or more years, making a placebo effect very unlikely. In addition, the fact that non-responders did not exhibit significant improvement during the follow up period makes it very unlikely the improvements observed in the 
responder group were due to the natural course of the condition. The favourable response to HT reported here is unlikely to be influenced to any great extent by the fact that treatment was at a specialist centre providing this treatment, since independent studies at other centres have also shown beneficial results with HT. ${ }^{10-12}$ Therefore, we believe our results could be generalised to the treatment of IBS patients in general. Out of all the pre-HT measures and demographic features examined with regression analysis, only gender was significantly associated with responder status, with males being more likely to be non-responders and this is in keeping with our previous observations. ${ }^{13}$

Very little deterioration occurred in IBS symptoms over time, although some of the other measures, such as certain extra-colonic symptoms, quality of life, and scores for anxiety and depression, did show some attrition, but most of these were still better at follow up than before treatment. Quality of life and psychological well being are influenced by many other factors such as life events, irrespective of gastrointestinal problems and it is possible that any of these could have been operating within an individual since treatment, thus contributing to the change in these scores. Anxiety and depression scores were significantly lower both post-HT and at follow up in both responders and non-responders, although the proportion of patients who could be classified as significantly anxious or depressed was only lower in responders. This would suggest that even though nonresponders did not improve in terms of IBS symptoms, they did derive some benefit psychologically. This is unlikely to be an effect of taking anxiolytics or antidepressants, since only a relatively small proportion of these non-responders were taking such medication after treatment. It is also interesting to note that nearly all patients considered the hypnotherapy to have been worthwhile, so that even non-responders felt that they had benefited in some way even though their symptoms had not altered with treatment.

Maintained improvement of symptoms did not appear to have any association with whether or not or how often responders continued to practise hypnotherapeutic techniques following treatment, although only $15 \%$ of responders discontinued practice. The reason for this somewhat unexpected finding is not readily apparent and does raise the question whether continued practice is necessary for any particular individual to maintain benefit. It may suggest that those who did well with HT yet did not continue to practise overtly have developed the ability to continue using their hypnotic skills at a subconscious level.

In conclusion, this study confirms that the beneficial effects of HT are long lasting, with continued improvement in symptoms, thus giving patients better control over their condition, although it may be less useful for male patients. A potential criticism for the use of hypnotherapy as a treatment for IBS has been that it is costly to provide because of the demands on therapists' time. However, because of its sustained effects in the majority of patients, costs of treatment could be rapidly offset by the ensuing reduction in cost of medication and other healthcare demands.

\section{APPENDIX 1 \\ IBS QUESTIONNAIRE ${ }^{14}$}

Items in the questionnaire were scored using a visual analogue scale of $0-100 \mathrm{~mm}$. Increased severity of IBS symptoms and extra-colonic features was indicated by a higher score, whereas increased impairment of quality of life measures was denoted by a lower score. Overall scores were calculated as the sum of the following individual items, adjusted as necessary to give a maximum score as indicated:
Overall IBS score (maximum 500; sum of 5 items): pain severity, pain frequency, distension, bowel habit dissatisfaction, life interference

Overall extra-colonic score (maximum 500; sum of 10 items $\div 2$ ): nausea/vomiting, early satiety, headaches, backache, excess wind, heartburn, bodily pains, urinary symptoms, thigh pain, lethargy.

\section{QUALITY OF LIFE}

The value of each quality of life measure was derived from the mean of the individual items shown indicated in parentheses, to give a maximum score of 100 .

- Psychic well being (coping with problems, confidence, usefulness, security)

- Physical well being (sleep, energy levels, aches and pains, feeling physically well)

- Mood (irritability, worrying, hopefulness, enjoyment of life)

- Locus of control (feeling in control of life, helplessness, ability to make decision)

- Social/relationship (relationships with family/partner, ability to maintain friendships, inferiority, feeling wanted, enjoyment of leisure).

\section{APPENDIX 2}

\section{SUBJECTIVE ASSESSMENT QUESTIONNAIRE (SAQ)}

This is a simple questionnaire devised in the Unit asking patients to rate the effects of HT on symptoms, consultation rates and use of medication etc, using Likert-type responses, as shown below.

\section{IBS symptoms}

(i) Compared with how you felt before HT, how would you rate your symptoms were at the end of the course of HT?

- very much better, moderately better, slightly better, about the same, slightly worse, moderately worse, very much worse

(ii) Compared with how your symptoms were at the end of the course of HT, would you say that since completing treatment your symptoms have:

- continued to: improve much more, moderately more, a little more; remained about same as at end of HT;

- gone: slightly worse, moderately worse, much worse.

\section{Consultation rates}

Since HT, have you had to consult:

(a) your GP (b) a hospital consultant about IBS symptoms:

- more often, about the same, less often (than before)

(c) your GP about other symptoms:

- more often, about the same, less often (than before).

\section{Use of medication}

(a) Do you currently take medication for IBS symptoms? Yes, No (If yes, please specify)

(b) Do you take medication:

- more often, about the same, less often (than before) 
(Information on any medication taken before starting the course of HT was recorded at the time.)

\section{Continued practice}

Do you still practise HT techniques? Yes, No If so, do you practise:

(a) using tape, on own

(b) daily, several times/week, several times/month, rarely

\section{Did you consider the course of HT to be worthwhile? Yes, No}

\section{6. (a) Have you tried any other treatment/therapy} since HT to relieve IBS symptoms? Yes, No

(b) If so, what?

(c) Did you find this to be helpful? Yes, No

\section{ACKNOWLEDGEMENTS}

We wish to thank the other staff of the Hypnotherapy Unit who treated some of the patients used in this study: Mrs P Cooper, Mrs P Cruickshanks, Mrs J Randles, and Mrs V Whelan. We also thank Mrs Julie Morris, Head of Medical Statistics, University Hospital of South Manchester, for help with statistical analysis.

\section{Authors' affiliations}

W M Gonsalkorale, V Miller, A Afzal, P J Whorwell, Department of Medicine, University Hospital of South Manchester, Manchester, UK

\section{REFERENCES}

1 Whorwell PJ, McCallum M, Creed FH, et al. Non-colonic features of irritable bowel syndrome. Gut 1986;27:37-40.
2 Switz DM. What the gastroenterologist does all day. A survey of a state society's practice. Gastroenterology 1976;70:1048-50

3 Harvey RF, Salih SY, Read AE. Organic and functional disorders in 2000 gastroenterology outpatients. Lancet 1983;i:632-4.

4 Drossman DA, Li Z, Andruzzi E, et al. U.S. householder survey of functional gastrointestinal disorders: prevalence, sociodemography and health impact. Dig Dis Sci 1993;38:1569-80

5 Maxton DG, Whorwell PJ. Use of medical resources and attitudes to health care of patients with 'chronic abdominal pain'. Br J Med Econ 1992;2:75-9.

6 Talley NJ, Gabriel SE, Harmsen WS, et al. Medical costs in community subjects with irritable bowel syndrome. Gastroenterology 1995; 109:1736-41.

7 Whorwell PJ, Prior A, Faragher EB. Controlled trial of hypnotherapy in the treatment of severe refractory irritable bowel syndrome. Lancet 1984;ii:1232-4.

8 Whorwell PJ, Prior A, Colgan SM. Hypnotherapy in severe irritable bowel syndrome: further experience. Gut 1987;28:423-5.

9 Houghton LA, Heyman DJ, Whorwell PJ. Symptomatology, quality of life and economic features of irritable bowel syndrome-the effect of hypnotherapy. Aliment Pharmacol Ther 1996;10:91-5.

10 Harvey R, Hinton RA, Gunary RM, et al. Individual and group hypnotherapy in the treatment of refractory irritable bowel syndrome. Lancet 1989;i:424-5.

11 Galovski TE, Blanchard EB. The treatment of irritable bowel syndrome with hypnotherapy. Appl Psychophysiol Biofeedback 1998;23:219-32.

12 Palsson OS, Turner MJ, Johnson DA, et al. Hypnosis treatment for severe irritable bowel syndrome-investigation of mechanisms and effects on symptoms. Dig Dis Sci 2002;47:2605-14.

13 Gonsalkorale WM, Houghton LA, Whorwell PJ. Hypnotherapy in irritable bowel syndrome: a large-scale audit of a clinical service with examination of factors influencing responsiveness. Am J Gastroenterol 2002;97:954-61.

14 Francis CY, Morris J, Whorwell PJ. The irritable bowel severity scoring system: a simple method of monitoring irritable bowel syndrome and its progress. Aliment Pharmacol Ther 1997;11:395-402.

15 Zigmond AS, Snaith RP. The Hospital Anxiety and Depression scale. Acta Psychiatr Scand 1983:67:361-70.

16 Veldhuzen van Zanten $S$, Talley N, Bytzer $P$, et al. Design of treatment trials for functional bowel disorders. Gut 1999;45:1169-77.

17 Spiller RC. Problems and challenges in the design of irritable bowel syndrome clinical trials: experience from published trials. Am J Med 1999; 107:91-7S. 